\title{
MR-guided blood-brain barrier opening induced by rapid short- pulse ultrasound in non-human primates
}

\author{
Hui Zhou ${ }^{1,2}$, Yang Liu ${ }^{3}$, Xiaojing Long ${ }^{3}$, Yangzi Qiao ${ }^{1}$, Jo Lee ${ }^{1}$, Xin Liu ${ }^{1,4}$, Hairong Zheng ${ }^{1}$, Chao Zou ${ }^{1,4}$ \\ ${ }^{1}$ Paul C. Lauterbur Research Center for Biomedical Imaging, Shenzhen Institutes of Advanced Technology, Chinese Academy of Sciences, Shenzhen, \\ China; ${ }^{2}$ The Shenzhen College of Advanced Technology, University of Chinese Academy of Sciences, Shenzhen, China; ${ }^{3}$ Research Center for \\ Medical AI, Shenzhen Institutes of Advanced Technology, Chinese Academy of Sciences, Shenzhen, China; ${ }^{4}$ Key Laboratory for Magnetic Resonance \\ and Multimodality Imaging of Guangdong Province, Shenzhen, China
}

Correspondence to: Dr. Chao Zou; Dr. Hairong Zheng. Paul C. Lauterbur Research Center for Biomedical Imaging, Shenzhen Institutes of Advanced, Technology, Chinese Academy of Sciences, 1068 Xueyuan Avenue, Shenzhen University Town, Shenzhen, China.

Email: chao.zou@siat.ac.cn; hr.zheng@siat.ac.cn.

Background: Opening the blood-brain barrier (BBB) with focused ultrasound and microbubbles (MBs) has potential use in non-invasive targeted therapy for central nervous system (CNS) diseases. Rapid short-pulse (RaSP) ultrasound with a microsecond sequence has been proposed as a minimally disruptive and efficient method for opening the BBB. This work aimed to test the feasibility and safety of BBB opening in a nonhuman primate model using combined RaSP ultrasound sequence and MBs.

Methods: The BBB of 2 rhesus macaques were opened with RaSP and the commonly used 10 millisecond long pulse (LP), combined with microbubble (SonoVueTM, $0.2 \mu \mathrm{L} / \mathrm{g}$ ) injection in a bolus. The transducer's central frequency was $300 \mathrm{kHz}$, and the acoustic pressure was set to $0.56 \mathrm{MPa}$ calibrated in water. The BBB opening procedure was guided and evaluated with contrast-enhanced magnetic resonance imaging. The relative signal enhancement was compared between RaSP and LP sonication. T2-weighted fast-spin echo (FSE) and T2*-weighted gradient echo (GRE) sequences were scanned to evaluate edema and microbleeding at the end of the procedure.

Results: The relative signal enhancement was significantly higher $(\mathrm{P}<0.01)$ in the focal area compared to a similar area of the opposite hemisphere at all time points after sonication in each monkey, indicating the successful opening of the BBB. The relative signal enhancement in RaSP reached more than $60 \%$ of that with LP in our experiment, while the energy deposition was only $6 \%$ of LP. No edema or hemorrhage was found on magnetic resonance images after RaSP.

Conclusions: Combined RaSP ultrasound and MBs for the BBB opening is a practical method in large animal models.

Keywords: Magnetic resonance imaging (MRI); rapid short-pulse ultrasound (RaSP ultrasound); blood-brain barrier opening (BBBO); non-human primates

Submitted Sep 08, 2020. Accepted for publication Jan 15, 2021.

doi: 10.21037/qims-20-1047

View this article at: http://dx.doi.org/10.21037/qims-20-1047

\section{Introduction}

The blood-brain barrier (BBB) is a selectively permeable boundary separating the parenchyma of the central nervous system (CNS) from systemic circulation (1). The BBB keeps the microenvironment of the CNS stable while limiting the passage of most small pharmaceutical molecules and nearly all large molecules, such as monoclonal antibodies, gene-therapy drugs, and recombinant proteins (2). Various diagnostic agents and drugs have been developed to treat CNS diseases; however, the intact BBB can prevent these 
agents, whether taken intravenously or orally, from reaching the CNS in effective amounts. Therefore, methods to permit the passage of desired substances in effective amounts through the BBB are needed.

Several methods have been studied to overcome the BBB, including lipophilic chemical modification of agents or the use of biological transporters. However, these measures have low spatial specificity unless intra-arterial infusion or direct transcranial administration are used, which are not ideal for targeted drug delivery (3). Temporarily opening the BBB for drug delivery with low-intensity pulsed ultrasound (4-6) combined with microbubbles (MBs) has shown promise in preclinical studies in animal models and even in human subjects (5,7-14). Ultrasound parameters, $M B$ types, and the dose applied are the key determinants of BBB opening's safety and effectiveness $(1,9,15,16)$. Magnetic resonance imaging (MRI) is widely adopted for feasibility and safety verification in these studies. Hynynen et al. (7) and McDannold et al. (8) applied low-intensity focused ultrasound in conjunction with intravenously administered MBs (Optison, diameter: $2.0-4.5 \mu \mathrm{m}, 0.05 \mathrm{~mL} / \mathrm{kg}$ ) for targeted $\mathrm{BBB}$ opening in rabbits under the guidance of MRI. The acoustic pressures varied from 1 to $4.7 \mathrm{MPa}$ at $1.63 \mathrm{MHz}$, and burst length varied from 10 to $100 \mathrm{~ms}$ with $1 \mathrm{~Hz}$ repetition in these studies, and the $\mathrm{BBB}$ recovered within 24-48 hours with no neuronal damage below $1 \mathrm{MPa}$. Brain tissue damage was found above $2.3 \mathrm{MPa}$, while the rabbits did not show any neurological symptoms. Samiotaki et al. (9) and Downs et al. (10) conducted quantitative studies with MRI on focused ultrasound-induced $\mathrm{BBB}$ opening in mice (acoustic pressures $0.30-0.60 \mathrm{MPa}$ at $1.5 \mathrm{MHz}, 67 \mu \mathrm{s}$ burst length, $10 \mathrm{~Hz}$ repetition; MBs diameter: $1-8 \mu \mathrm{m}$, concentration $1 \mathrm{~mL} / \mathrm{kg}$ ) and in the longterm estimation of safety in non-human primates (acoustic pressures $200-400 \mathrm{kPa}$ at $500 \mathrm{kHz}, 10 \mathrm{~ms}$ burst length, $2 \mathrm{~Hz}$ repetition; MBs diameter: $4-5 \mu \mathrm{m}$, concentration $0.1 \mathrm{~mL} / \mathrm{kg}$ ). The authors found that the $\mathrm{BBB}$ remained open for 24 hours or sometimes for several days in mice due to the possible vascular damage under higher acoustic pressures or larger MBs. In the primate study with a cognitive task, primates' task-reaction time increased significantly on the day of sonication, without long-term negative physiological or neurological effects, and returned to baseline within 4-5 days. Chai et al. (17) opened the BBB of rats with a $400 \mathrm{kHz}$ ultrasound transducer and measured the kinetics of permeability changes with dynamic contrastenhanced MRI. The kinetics recovered to baseline within 24 hours. Recently, BBB opening with MR-guided focused ultrasound was investigated in patients being treated for neurological diseases (11-13).

Despite the BBB opening reports with the combination of ultrasound and MBs, the potential for side-effects, such as neuronal damage, red blood cell extravasation, and neuroinflammation, should be assessed before this technique is applied clinically $(12,13,16,18)$. Efforts have been made to improve these methods' safety by optimizing the acoustic and MB parameters to shorten the recovery time of the BBB and reduce albumin infiltration into the brain. Sonication with short pulses $(<1 \mathrm{~ms})$ is reported to be sufficient for BBB opening with much less energy deposition. Choi et al. disrupted the BBB of mice using a $33 \mu$ s burst length at $1.5 \mathrm{MHz}$ with $10 \mathrm{~Hz}$ repetition (19). O'Reilly et al. (20) induced $\mathrm{BBB}$ disruption in rats with a single $3 \mu$ s pulse burst (at $1.18 \mathrm{MHz}$ with $1 \mathrm{~Hz}$ repetition under $0.54 \mathrm{MPa}$ ). Samiotaki et al. (21) found that $67 \mu$ s pulse length with $10 \mathrm{~Hz}$ repetition could also cause BBB opening in mice under $0.45 \mathrm{MPa}$ acoustic pressure, and the permeability change, opening volume, and reversal time were comparable to those of long pulses (LP) (>1 ms) under lower acoustic pressure.

Recently, Morse et al. (22) reported that a rapid shortpulse $(\mathrm{RaSP})$ ultrasound sequence $(5 \mu$ sursts per $0.8 \mathrm{~ms}$, continuously repeated 13 times every 2 seconds, acoustic power $0.35 \mathrm{MPa})$ with SonoVue MBs $(5 \mu \mathrm{L} / \mathrm{g}$ injections in vein) was efficient for drug delivery with minimal BBB disruption in mice. Their study showed that the drugs were more uniformly delivered into the brain than the traditional $10 \mathrm{~ms}$ LP (LP10). The duration of the permeability change was reduced to less than 10 minutes by RaSP. The amount of albumin released into the brain was 3.4-fold less than LP10, while the dextran delivery dosage was not significantly reduced. Although drug delivery efficiency in the long-term period of RaSP needs further optimization, the features of a uniform delivery profile and shorter BBB recovery time of RaSP are attractive to diagnosing and treating neurological diseases.

To the best of our knowledge, RaSP induced-BBB opening has only been reported in rodent studies. This work aims to verify the feasibility and safety of BBB opening with RaSP in a non-human primate model. Contrast-enhanced MRI was used to evaluate BBB opening quantitatively in both RaSP and LP trials. Traditional T2weighted $(\mathrm{T} 2 \mathrm{w})$ and $\mathrm{T} 2{ }^{*} \mathrm{w}$ images were used to detect edema and hemorrhage. The results showed that RaSP achieved the desired opening of the BBB without adverse side effects under current ultrasound and MB parameters in 


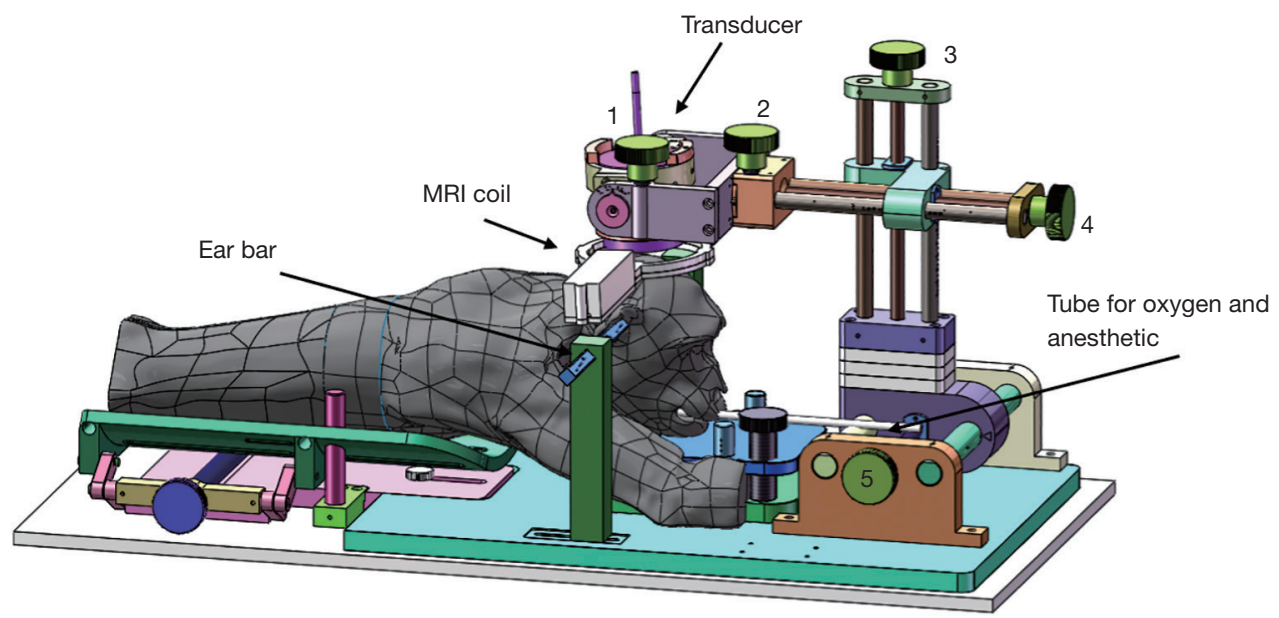

Figure 1 Experimental setup. The animal was prone on an MR-compatible stereotaxic frame and fixed by 2 ear bars. The ultrasound transducer $(300 \mathrm{kHz})$ was fixed on a positioning system with 5 degrees of freedom (1 and 2 for rotational motions and 3, 4, 5 for translational motions) and coupled to the animal with degassed water. A single-element radiofrequency coil was inserted between the transducer and the animal's head to receive MR signals. MRI, magnetic resonance imaging.

a monkey model.

\section{Methods}

\section{Animal preparation}

The Institutional Ethical Committee approved all animal procedures of Animal Experimentation of Shenzhen Institutes of Advanced Technology, Chinese Academy of Sciences. The experiments (Certificate number: originalSIAT-IRB-170425-YGS-ZHR-A0094-2, amended-SIATIACUC-20200417-ZKYSZXJJSYJY-YB-ZHR-2-04) were carried out strictly following governmental and international guidelines on animal experimentation. All efforts were made to minimize the number of animals used and their suffering during experiments, according to the request of Biosafety and Animal Ethics. Two male rhesus macaques (M21-monkey 21; M22-monkey 22) weighing 5.8 and $6.9 \mathrm{~kg}$ were used. The animals were allowed only water $12 \mathrm{~h}$ before the experiments. Before the experiments, the study animal was intramuscularly injected with atropine $(0.02-0.04 \mathrm{mg} / \mathrm{kg})$ in their cage. Ten minutes later, telazol $(5 \mathrm{mg} / \mathrm{kg})$ was administered by intramuscular injection for temporary anesthesia. After the monkey was fully anesthetized, it was transported to the animal preparation room, and its head was shaved. The monkey was fixed by 2 ear bars in a stereotaxic frame on the MR scanning table and intubated for anesthesia during the experiment. The animal was covered by a blanket with circulating warm water to maintain its body temperature. Vital signs were monitored with an MRI-compatible patient monitor (Precess 3160, Invivo, Philips) and manually recorded. The interval between experiments was approximately 2 weeks for each monkey. The experimental setup is shown in Figure 1.

\section{Ultrasound system}

A custom-built, single-element, spherical focused ultrasound transducer with a diameter of $50 \mathrm{~mm}$ and a geometric focal length of $55 \mathrm{~mm}$ was used for sonication. The central frequency of the transducer was $300 \mathrm{kHz}$. The transducer was implanted in an ultrasound coupler filled with degassed water and fixed on the positioning system with 5 degrees of freedom (2 rotational motions and 3 translational motions). The transducer's acoustic pressure was calibrated by hydrophone (Precision Acoustics Ltd., Dorchester, UK) in water, as illustrated in Figure S1. The transducer was driven by a custom-built power amplifier with a fixed gain of $50 \mathrm{~dB}$, as illustrated in Figure S2.

\section{Sonication protocol}

The timing diagrams of the schemes of the 2 kinds of RaSP sequences and an LP sequence were compared, as presented in Figure 2. The pulse repetition frequency (PRF) of the ultrasound was $1 \mathrm{~Hz}$, the burst length was $10 \mathrm{~ms}$ for the LP 

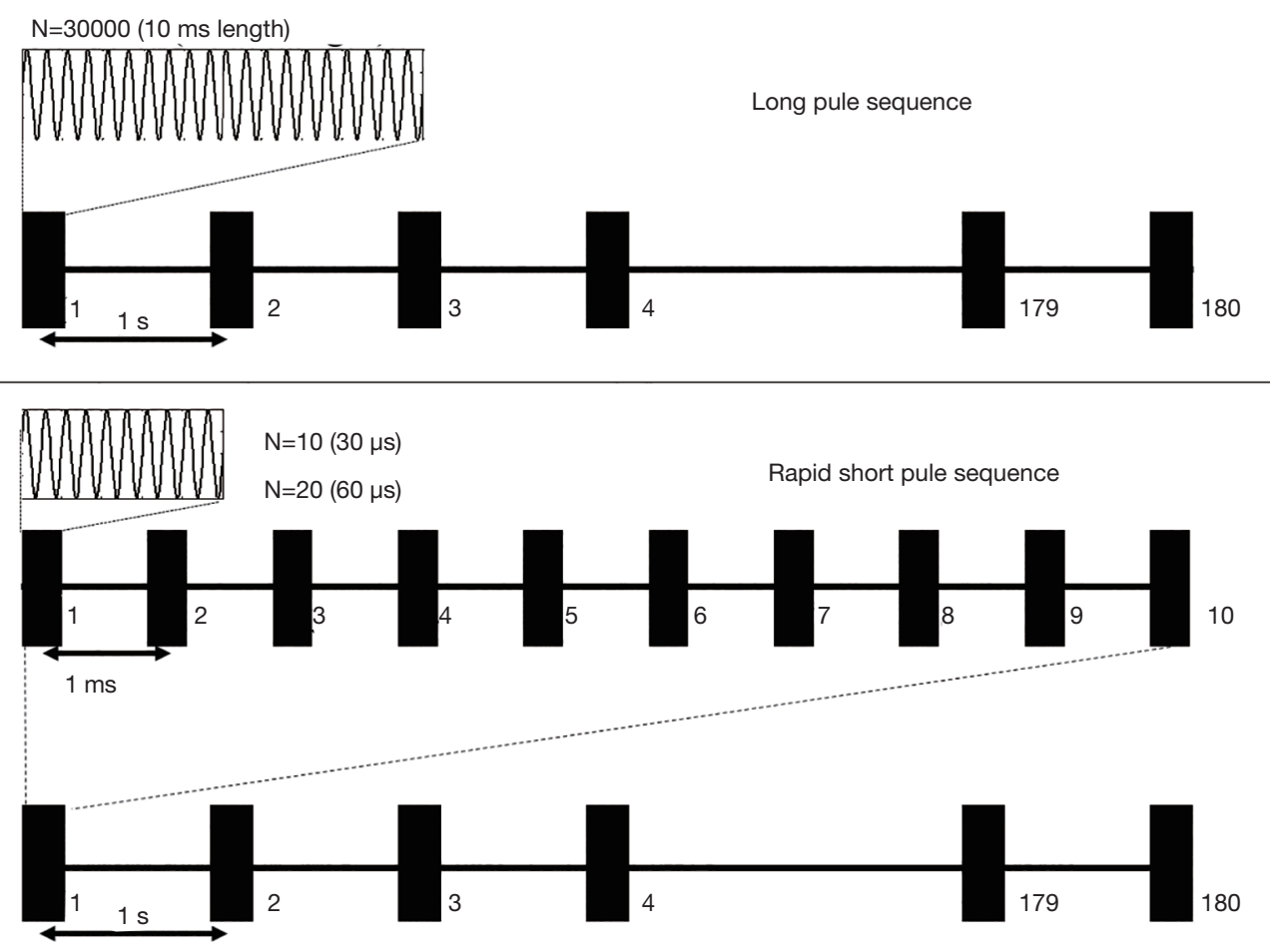

Figure 2 Ultrasound sequences. A $10 \mathrm{~ms}$ length pulse (LP10) was partitioned into 10 periods of $1 \mathrm{~ms}$ each. Each period contained only $10(\mathrm{RaSP} 30)$ or 20 (RaSP60) pulses of sine waves with $300 \mathrm{kHz}$. The pulse repetition frequency of sonication was $1 \mathrm{~Hz}$, the peak negative acoustic pressure of all sequences was $0.56 \mathrm{MPa}$ calibrated in water, and the corresponding mechanical index (MI) was 1.02 in water. N, the number of ultrasound waves.

(LP10), and $300 \mu$ s (RaSP 30) or $600 \mu$ s (RaSP 60) for the RaSP sequences. The 10 ms burst length in LP was further partitioned into 10 periods, and only 10 or 20 pulses were applied (equal to $30 \mu$ s or $60 \mu$ pulse length) during each $1 \mathrm{~ms}$ period, making the total duty cycle $3 \%$ or $6 \%$ of LP sequences. The total duration of the ultrasound was $180 \mathrm{~s}$, and the peak negative acoustic pressure was set to $0.56 \mathrm{MPa}$ calibrated in water for both sequences. The relative mechanical index (MI) was 1.02 in water.

\section{BBB opening procedure}

The BBB opening procedure was guided and evaluated with a 3.0 T MRI scanner (uMR790, Shanghai United Imaging Healthcare, Shanghai, China). Before sonication, $\mathrm{T} 1, \mathrm{~T} 2$, and $\mathrm{T} 2{ }^{*} \mathrm{w}$ images (imaging protocol is presented in Table 1) were obtained for treatment planning and used as a baseline. The prepared animal was sonicated with the pulsed ultrasound for $3 \mathrm{~min}$ after the MBs (SonoVue ${ }^{\mathrm{TM}}$, Bracco International, Amsterdam, The Netherlands), with mean diameters approximately $2.5 \mu \mathrm{m}$ and concentration
$1 \times 10^{8}$ to $5 \times 10^{8} \mathrm{MBs} / \mathrm{mL}$, were injected through the femoral vein. At the end of sonication, $1 \mathrm{~mL}(0.15-0.17 \mathrm{~mL} / \mathrm{kg}$ per monkey) gadopentetate dimeglumine (Gd-DTPA) was injected, and a series of T1w fast-spin echo (FSE) images with spectral attenuated inversion recovery (SPAIR) were acquired continuously to demonstrate the contrast enhancement of BBB opening. MBs and Gd-DTPA injection were followed by $1 \mathrm{ml}$ saline to flush the agent out of the retention needle. Finally, the T2w FSE and $\mathrm{T} 2{ }^{*} \mathrm{w}$ gradient echo (GRE) sequences were scanned to evaluate edema (23) and micro-bleeding (24) at the end of the procedure. $\mathrm{T} 1 \mathrm{w}$ fluid-attenuated inversion recovery (T1-FLAIR) was also used in some cases to identify the distribution of contrast enhancement with cerebral spinal fluid suppression. The experiments were classified into 5 groups (Figure 3) according to the ultrasound protocols: G1, RaSP for $300 \mu s ;$ G2, RaSP for $600 \mu s ;$ G3, LP for $10 \mathrm{~ms}$; G4, sequential RaSP for $300 \mu \mathrm{s}$ and RaSP for $600 \mu \mathrm{s}$; G5, sequential $300 \mu \mathrm{RaSP}$ and $10 \mathrm{~ms}$ LP ultrasound. Each animal received all 5 experimental protocols. In G4 and G5 studies, the sonication and T1w FSE acquisition were 
Table 1 MRI protocols

\begin{tabular}{|c|c|c|c|c|}
\hline Variables & T1-FSE & T1-FLAIR & T2-FSE & T2*-GRE \\
\hline TE (ms) & 11 & 11 & 75 & 20 \\
\hline $\mathrm{FA}\left({ }^{\circ}\right)$ & 90 & 90 & 90 & 30 \\
\hline FOV $(\mathrm{mm})$ & 120 & 120 & 120 & 120 \\
\hline Slices (mm) & 2.0 & 2.0 & 2.0 & 2.0 \\
\hline Slices gap & $20 \%$ & $20 \%$ & $20 \%$ & $20 \%$ \\
\hline Inversion time (ms) & - & 870 & - & - \\
\hline Echo train length & 3 & 5 & 16 & - \\
\hline
\end{tabular}

MRI, magnetic resonance imaging; TR, time of repetition; TE, time of echo; FA, flip angle; FOV, field of view; GRE, gradient-echo; FLAIR, fluid-attenuated inversion recovery.

G1,G2,G3:

\begin{tabular}{|c|c|c|c|}
\hline \multicolumn{3}{c}{3 Minutes } & More Than 90 Minutes \\
\hline $\begin{array}{c}\text { T1/T2/T2 } x \\
\text { Baseline }\end{array}$ & MB+ & Repeated & $\begin{array}{c}\text { T2/T2 } x \\
\text { Detection }\end{array}$ \\
\hline
\end{tabular}

\begin{tabular}{|c|c|c|c|c|}
\hline \multirow[t]{4}{*}{ G4,G5: } & & 3 Minutes & More Than 90 Minutes & \\
\hline & $\begin{array}{l}\mathrm{T} 1 / \mathrm{T} 2 / \mathrm{T} 2 \times \\
\text { Baseline }\end{array}$ & $\begin{array}{c}\mathrm{MB}+ \\
\mathrm{US}+\mathrm{Gd}\end{array}$ & $\begin{array}{c}\text { Repeated } \\
\text { T1w images }\end{array}$ & $\begin{array}{c}\text { T2/T2x } \\
\text { Detection }\end{array}$ \\
\hline & & 3 Minutes & More Than 90 Minutes & \\
\hline & & $\begin{array}{c}\mathrm{MB}+ \\
\mathrm{US}+\mathrm{Gd}\end{array}$ & $\begin{array}{l}\text { Repeated } \\
\text { T1w images }\end{array}$ & $\begin{array}{c}\text { T2/T2x } \\
\text { Detection }\end{array}$ \\
\hline
\end{tabular}

Figure 3 Procedures for the study groups. The ultrasonic protocols in G1, G2, and G3 were RaSP30, RaSP60, and LP10, respectively. In G4, RaSP30 was followed by RaSP60. In G5, RaSP30 was followed by LP10. MB, microbubbles; US, ultrasound; Gd, gadopentetate dimeglumine.

repeated once.

$$
S_{N}(t)=\frac{S(t)}{N(t)}
$$

\section{Data processing}

A reference region of interest (ROI) was drawn in the coupling water area, and the averaged signal intensity over the reference ROI was used to normalize the whole image to avoid signal fluctuation: where $S(t)$ is the T1w image acquired at time t, $N(t)$ is the averaged signal intensity over the reference ROI, and $S_{N}(t)$ is the resultant normalized T1w image at time $t$.

Signal enhancement maps at each time point were defined by the relative signal change against the image 

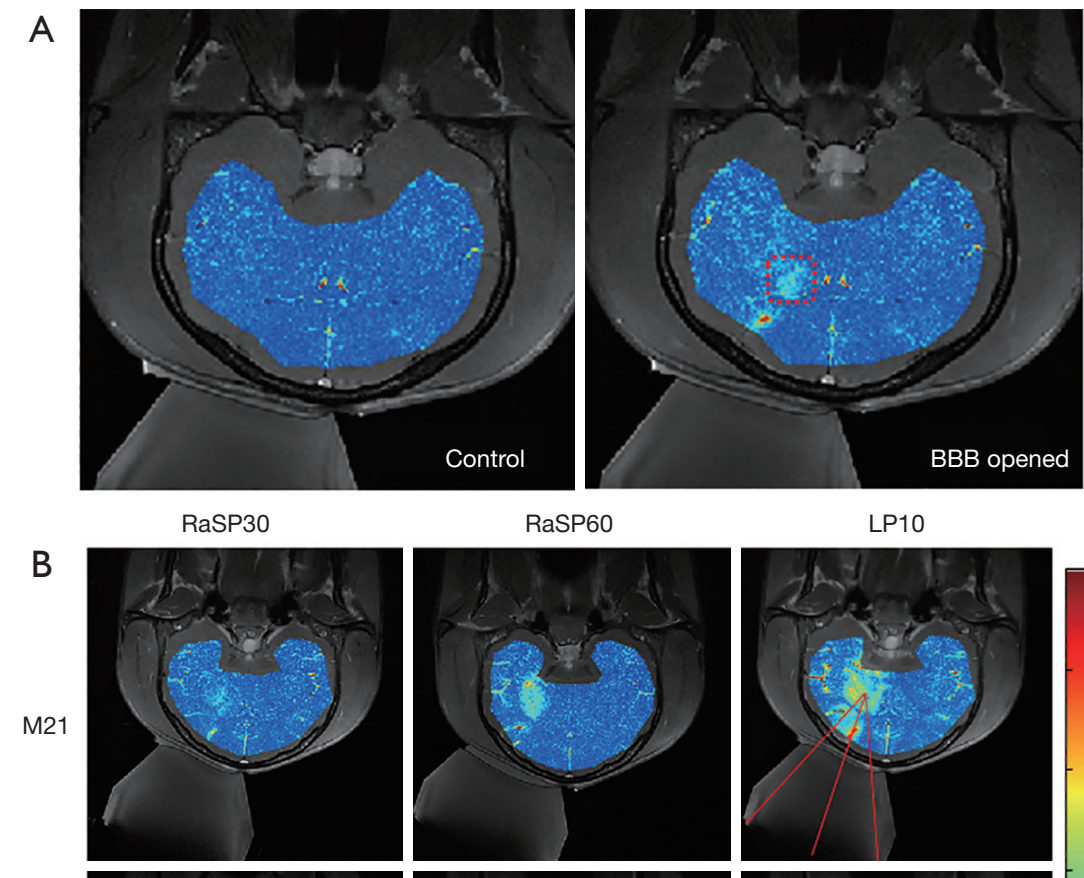

RaSP60

LP10
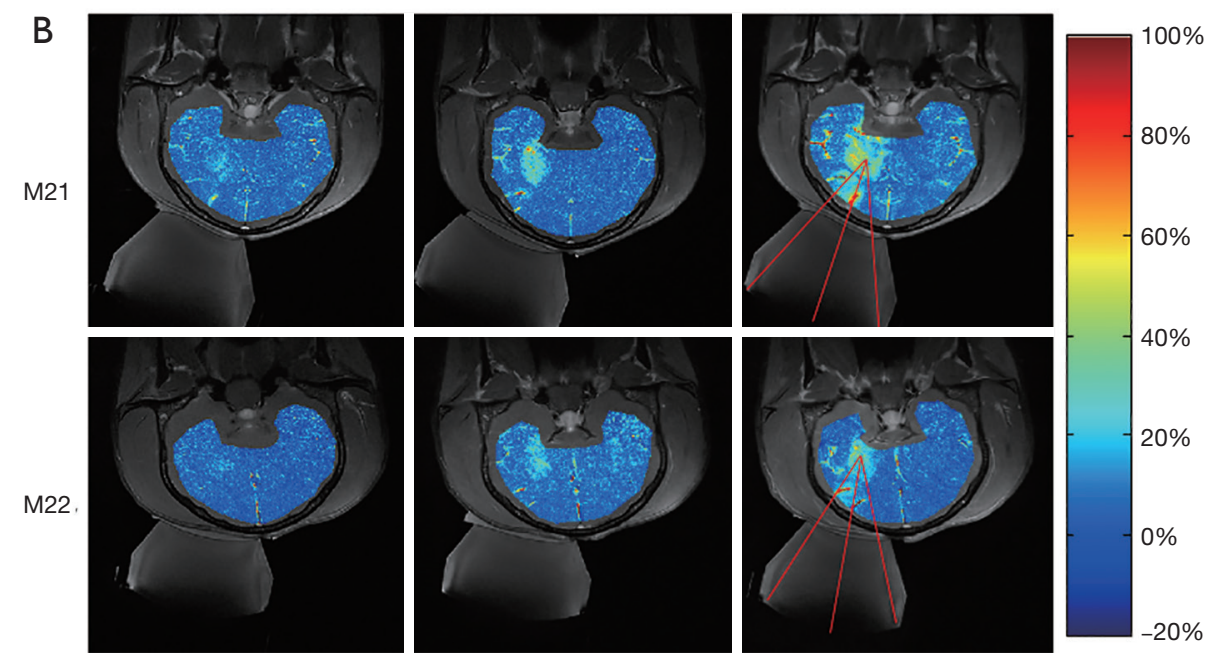

Figure 4 Relative signal enhancement maps overlapped with T1w images. (A) Relative signal enhancement maps for control (only Gd injection before RaSP60 sonication with MBs) and BBB opened (after RaSP60 sonication with MBs). Enhancement is indicated in a focal area (dotted rectangle). (B) Relative signal enhancement maps of M21 and M22 in all 3 protocols. The intersection of 3 red lines (representing the beam path) marks the geometric focus. The colorbar of the relative enhancement map is shown on the lower right-hand side for all images. BBB, blood brain barrier; M21, monkey numbered 21; M22, monkey numbered 22; RaSP30, rapid short pulse with 30 s burst sonication; RaSP60, rapid short pulse with $60 \mu$ s burst sonication; LP10, 10 ms long pulse sonication.

acquired before Gd-DTPA was injected:

$$
\Delta S_{N}(t)=\frac{S_{N}(t)-S_{N}(0)}{S_{N}(0)}
$$

\section{Statistical analysis}

Two ROIs were drawn on the T1w images. One was arranged around the ultrasound focal area $\left(\mathrm{ROI}_{\mathrm{F}}\right)$, and the other was positioned on the opposite hemisphere for comparison (ROIc). The temporal course of the relative signal enhancement was analyzed. The Student's $t$-test was performed on the relative signal enhancement between the 2 ROIs at different time points in each trial. The statistical analysis was performed to determine whether the contrast enhancement appeared in the sonicated area to check that the BBB was opened in each trial.

\section{Results}

\section{RaSP-induced BBB opening}

The feasibility of BBB opening by RaSP was demonstrated by contrast enhancement around the targeted focal area after ultrasound sonication in all experiments. The relative signal enhancement maps overlapped on the T1w images. Figure $4 A$ shows the relative signal enhancement maps before and after RaSP60 sonication. The dotted 


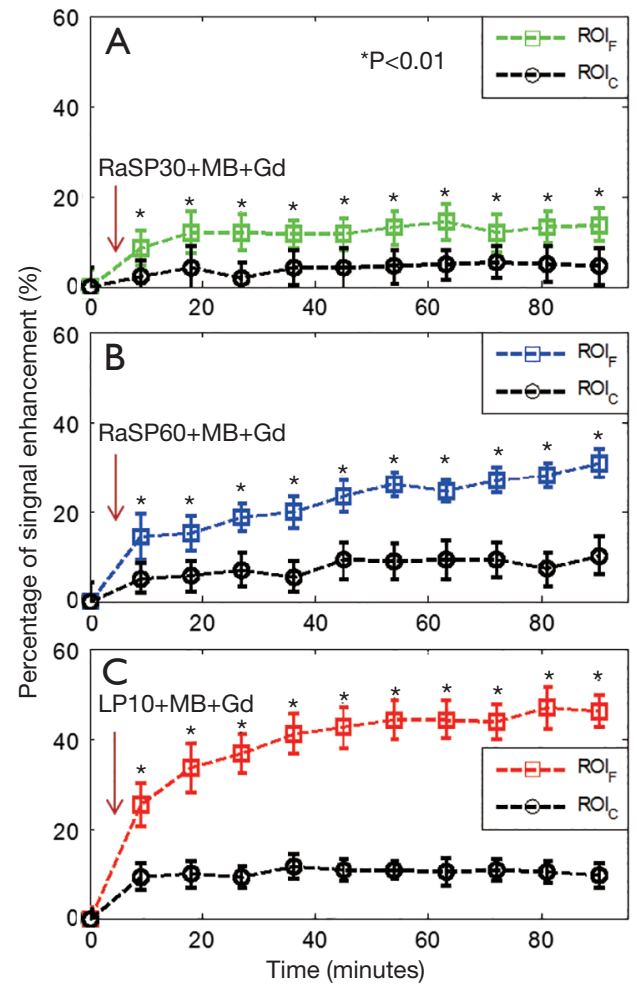

Figure 5 The relative signal enhancement curves after BBB opening with RaSP30, RaSP60, and LP10 in M21. The standard deviations of the relative signal enhancement inside the ROI are shown as the error bars at each time point. The differences between relative signal enhancement after sonication were all significant $\left({ }^{*}\right.$, represents $\mathrm{P}<0.01$ in Student's $t$-test) between pixels of $\mathrm{ROI}_{\mathrm{F}}$ and $\mathrm{ROI}_{\mathrm{C}}$ in the 3 protocols. RaSP30, Rapid short pulse with $30 \mu$ surst sonication; RaSP60, Rapid short pulse with $60 \mu$ surst sonication; LP10, $10 \mathrm{~ms}$ long pulse sonication; $\mathrm{MB}$, microbubble; $\mathrm{Gd}$, gadopentetate dimeglumine (Gd-DTPA); $\mathrm{ROI}_{\mathrm{F}}$, region of interest at focus; $\mathrm{ROI}_{\mathrm{C}}$, region of interest at the opposite hemisphere for comparison.

rectangle in the relative enhancement map after RaSP60 demonstrated observable signal change due to sonication. Figure $4 B$ compares the relative signal enhancement maps under RaSP30, RaSP60, and LP10 protocols in both monkeys. The contrast agent enhanced the area around the ultrasound focus, whereas no relative signal enhancement was evident in the opposite area in all protocols. The enhancement intensity and volume were larger under LP10.

\section{Temporal course of the relative signal enhancement after BBB opening}

The representative relative signal enhancement curves in
$\mathrm{ROI}_{\mathrm{F}}$ and $\mathrm{ROI}_{\mathrm{C}}$ of $\mathrm{M} 21$ are presented in Figure 5. Signal intensities slightly increased to a steady-state in the $\mathrm{ROI}_{\mathrm{C}}$, closely following Gd-DPTA injection, whereas they continuously increased in the $\mathrm{ROI}_{\mathrm{F}}$ up to $90 \mathrm{~min}$ after the Gd-DTPA injection in all protocols. For example, in the RaSP60 trial, as shown in Figure 5B, the relative signal enhancement in $\mathrm{ROI}_{\mathrm{C}}$ slightly increased to $5-8 \%$ during the whole observation time window, while the relative signal enhancement in $\mathrm{ROI}_{\mathrm{F}}$ continuously increased from $15 \%$ to $30 \%$ within 90 minutes. The student's t-test was performed between pixels of the 2 ROIs at different time points after sonication with MBs. The relative signal enhancement was significantly different in $\mathrm{ROI}_{\mathrm{F}}$ and $\mathrm{ROI}_{\mathrm{C}}(\mathrm{P}<0.01)$ at all time points after sonication in each monkey, indicating the successful opening of the BBB in these trials.

\section{Signal enhancement comparison}

To quantitatively compare the differences in BBB opening induced by the various protocols, we evaluated the relative signal enhancement in $\mathrm{ROI}_{\mathrm{F}}$ and $\mathrm{ROI}_{\mathrm{C}}$ for the 5 experimental groups. Three time points at which the 3 largest mean values occurred in $\mathrm{ROI}_{\mathrm{F}}$ were found for all curves. The average of the mean and standard deviation of these 3 time points are summarized in Table S1. The relative signal enhancement in $\mathrm{ROI}_{\mathrm{F}}$ after $\mathrm{BBB}$ opening was significantly higher than that in $\mathrm{ROI}_{\mathrm{C}}$ for all experimental groups $(\mathrm{P}<0.01)$. In all the study groups, the differences between relative signal enhancement in $\mathrm{ROI}_{\mathrm{F}}$ and $\mathrm{ROI}_{\mathrm{C}}$ were positively correlated with the burst length of pulses in each sonication protocol. The relative signal enhancement of the same protocol showed good consistency between different experimental groups in each animal, as illustrated in Table S1 and Figure 6. For example, in the $3 \mathrm{RaSP} 30$ trials in G1/G4/G5 on M21, the signal enhancement was $12.7 \% \pm 3.0 \%, 13.2 \% \pm 3.3 \%$, and $13.3 \% \pm 3.2 \%$, respectively. The relative signal enhancement in RaSP30 and RaSP60 reached more than $30 \%$ or $60 \%$ of that with LP 10 sonication in our experiment, respectively. Furthermore, the energy deposition in RaSP30 and RaSP 60 was only $3 \%$ and $6 \%$ of LP10, respectively. The relative signal enhancement in $\mathrm{ROI}_{\mathrm{F}}$ was $2-4$ folds higher compared to $\mathrm{ROI}_{\mathrm{C}}$ in $\mathrm{RaSP}$ sonications.

\section{Edema and hemorrhage detection}

T2 FSE and T2* GRE images were acquired with the same position, resolution, and slices as for the T1 FSE 


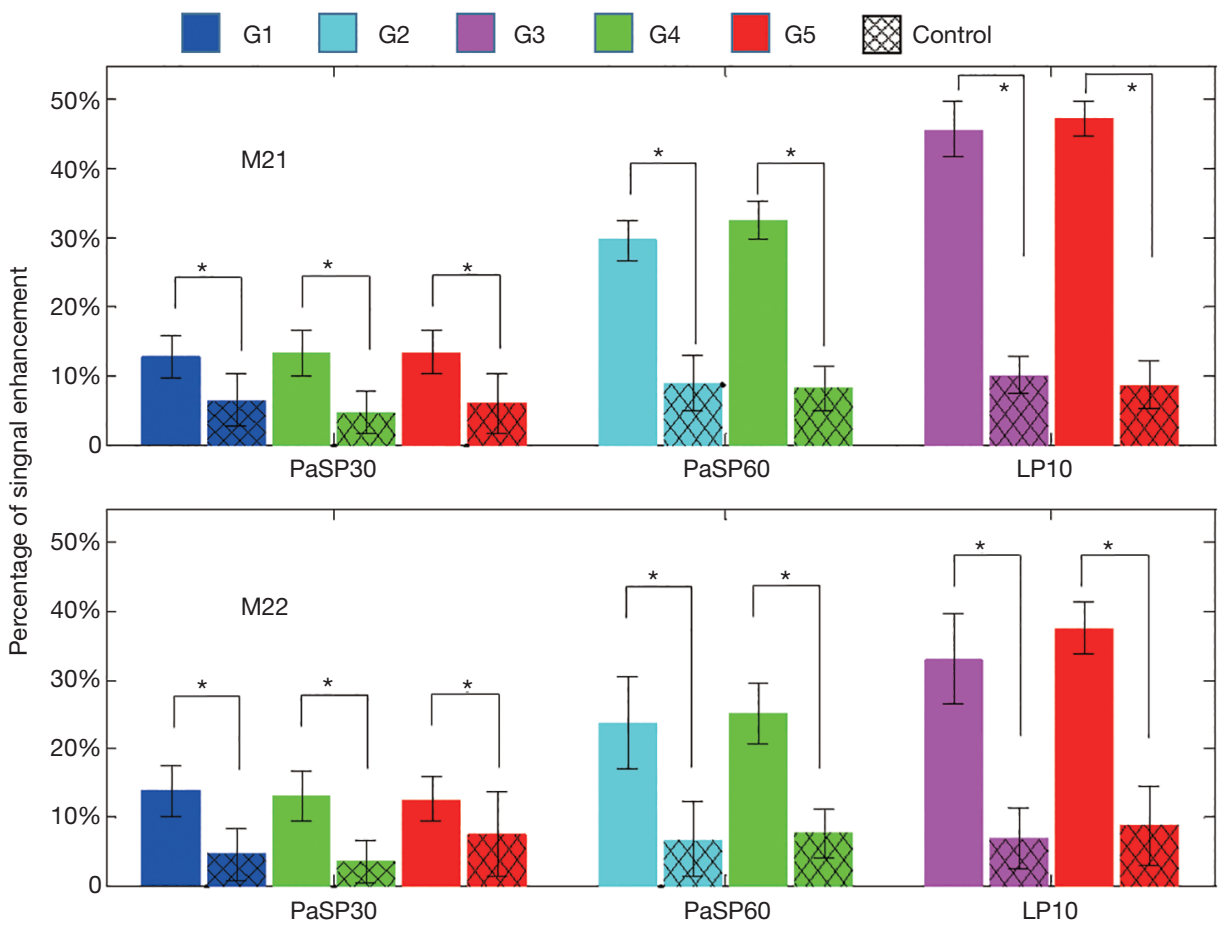

Figure 6 Bar plots of the relative signal enhancement for each protocol in the 2 monkeys. The colored bars and the hatch-filled bars represent the relative signal enhancement of ROIF and ROIC, respectively. *, represents $\mathrm{P}<0.01$. G1-G5 represent different experimental groups, controls represent the relative signal enhancement at the opposite hemisphere for each group. RaSP30, rapid short pulse with $30 \mu$ surst sonication; RaSP60, rapid short pulse with $60 \mu$ surst sonication; LP10, 10 ms long pulse sonication; M21, monkey numbered 21; M22, monkey numbered 22.

sequence to detect edema and micro-bleeding. There were no observable changes in the $\mathrm{T} 2 \mathrm{w} / \mathrm{T} 2{ }^{*} \mathrm{w}$ MRI images for RaSP30 and RaSP60. However, in the G5 experiment (RaSP30 followed by LP10 at the same location within 2 hours), hemorrhage in the sulcus caused by LP10 sonication was present in monkey M21, which was indicated by the hypointensity signal on $\mathrm{T} 2{ }^{*} \mathrm{w}$ imaging after LP10 (hypointensity signal was not present before LP10 sonication in Figure $7 A$ and appeared after LP10 as per the arrow in Figure 7B). The hypointensity disappeared 2 weeks later (arrow in Figure 7C). No enhancement was evident in the contrast-enhanced T1w images acquired 2 weeks after G5 sonication (Figure 7D).

\section{Discussion}

Studies over the past decades have found that 10 to $100 \mathrm{~ms}$ LP ultrasound combined with MBs can open the BBB without irreversible brain tissue damage in models from mice to non-human primates in vivo $(5,7,8,10-13)$. Also,
BBB opening with negligible disruption has been achieved with microsecond-level RaSP ultrasound in mice (22). However, the safety of ultrasound for BBB opening remains a concern for clinical application. Here, we documented the feasibility and safety of BBB opening with RaSP ultrasound in monkeys.

Contrast-enhanced T1w MRI is a commonly used (15,25-28) acceptable method for identifying BBB opening in large animals, such as monkeys. In these referenced reports, $10 \%$ relative signal enhancement was used to confirm the local BBB opening. Our study documented the feasibility of opening the BBB in non-human primates with RaSP. The relative signal enhancement was approximately $13 \%$ in RaSP30 and exceeded 23\% in RaSP60 in the sonicated area. The relative signal enhancement was positively correlated with the pulse length, which is consistent with previous studies (20). The concordance between the results obtained with the same ultrasound protocols across various experimental groups (G1 and G4/G5-RaSP30, G2 and G4RaSP60, G3 and G5-LP10) indicated high consistency of the 

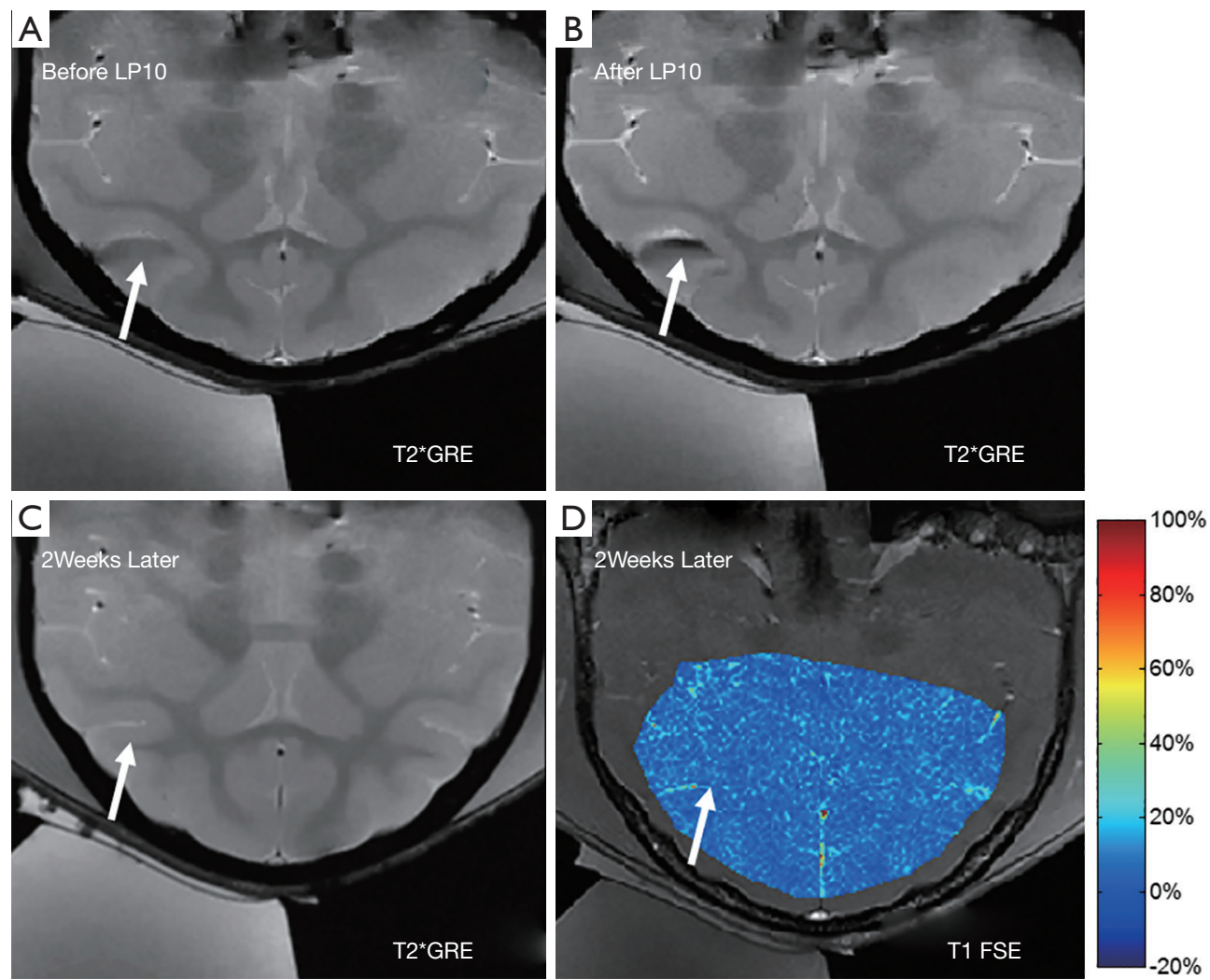

Figure 7 Hemorrhage detected in T2* ${ }^{*}$ GRE images after LP10 sonication in the G5 protocol in M21. (A) T2* ${ }^{*}$ GRE image acquired after RaSP30 and before LP10. (B) T2* ${ }^{*}$ GRE image acquired after LP10. Hypointensity in the sulcus (white arrow) indicates the presence of hemorrhage. (C) T2* ${ }^{*}$ GRE image acquired 2 weeks later, showing that the hypointensity disappeared. (D) Contrast-enhanced T1w image after Gd-DTPA injection (without ultrasound), with no local enhancement present, indicating that the BBB recovered to baseline. LP10, 10 ms long pulse sonication; FSE, fast-spin echo; GRE, gradient echo.

experiments in each monkey. However, there was a difference between the 2 monkeys for the same group of ultrasound variables, which may be due to differences in the skull geometry (27).

In monkeys, the BBB is restored within 2 to 4 days after being opened with LP (29). Shortened BBB recovery time is an attractive feature of RaSP compared with traditional LP. For example, in mice, permeability returned to control levels in less than 10 minutes with RaSP ultrasound (22). In our experiments in non-human primates, the duration of $\mathrm{BBB}$ opening may have been longer than desirable. In one experiment, the animal was injected again with Gd-DTPA (without ultrasound sonication) 90 minutes after a RaSP60 sonication. The relative signal enhancement continued to increase in the focal area, while it was unchanged in the contralateral control area, indicating that the BBB was still open (Figure S3). According to Samiotaki et al. (21,30),
BBB opening's reversal time is linearly correlated with the opening volume caused by a single foci sonication. O'Reilly et al. (31) claim that the key factors determining $\mathrm{BBB}$ recovery time are the acoustic pressure profile and MB parameters. We speculate that the shortening of $\mathrm{BBB}$ recovery time was not achieved due to the relatively higher acoustic pressure $(0.56 \mathrm{MPa}$ in water under $300 \mathrm{kHz})$ used in our study compared to Morse et al.'s work (0.35 MPa under $1 \mathrm{MHz}$ ) (22). The acoustic pressure profile of the focus was relatively broad in our study (focal area $6.9 \mathrm{~mm}$ $\times 6.9 \mathrm{~mm} \times 30 \mathrm{~mm}$ according to Figure S1). Moreover, the MBs $(100 \mu \mathrm{L}, 5 \mu \mathrm{L} / \mathrm{g}$ SonoVue) was injected intravenously over 30 seconds in their study, while MBs $(1 \mathrm{~mL}, 0.2 \mu \mathrm{L} / \mathrm{g}$ SonoVue) were injected intravenously in a bolus in our study, The different MB administration modes may also contribute to the differences in the experimental results. In future work, acoustic parameters (including 
transducer geometry, acoustic pressure, PRF, burst period/ length, etc.) and MB parameters (bubble size, dosage, and administration) need further optimization to achieve shorter recovery time, especially in large animal models.

We were unable to more thoroughly study the exact recovery time of $\mathrm{BBB}$ opening because of institutional review board regulations on animal care (the minimal interval allowed for the MR scan on one monkey was 14 days). However, we conducted the Gd-DTPA enhancement scans (without ultrasound) 2 weeks after opening to ensure that the BBB had recovered from the sonication, and no relative signal enhancement was present (Figure 7D). In the future, we will aim to shorten the interval for MRI scanning in 1 or 2 monkeys to obtain a more precise measure of the recovery time.

The $300 \mathrm{kHz}$ transducer was chosen after considering the skull penetration efficiency of the acoustic power in monkeys. One of the adverse effects of using low-frequency ultrasound is that the cavitation threshold is also lowered. Measuring passive acoustic emissions is a widely used method of monitoring MB-induced cavitation (12,13,32-34). Although we did not monitor passive cavitation in our experiments, the acoustic parameters $(300 \mathrm{kHz}, 0.56 \mathrm{MPa}$, and $1.02 \mathrm{MI}$ in water) and MBs $(0.20 \mathrm{~mL} / \mathrm{kg}$; SonoVue) were carefully selected following a study of BBB disruption in non-human primates with feedback control of $M B$ cavitation (14). In this previous study, mild BBB opening was achieved under acoustic pressure $697 \mathrm{kPa}(500 \mathrm{kHz} ; \mathrm{MI}=0.99$ calibrated in free water). Harmonic components in the relative spectrum were observed, while broadband and sub-harmonic components were not significant in the passive cavitation detector (PCD), indicating that the MBs underwent stable cavitation. $\mathrm{T} 2 \mathrm{w}$ images with no hemorrhage evidence also confirmed that the BBB opened safely under this acoustic pressure in their study. Our MI (1.02 in free water) was very close to this previously reported MI for mild BBB opening (697 kPa, MI =0.99 calibrated in free water), and our $\mathrm{MB}$ dose $(0.20 \mathrm{~mL} / \mathrm{kg}$; SonoVue $)$ was slightly lower $(0.30 \mathrm{~mL} / \mathrm{kg}$; SonoVue). We also confirmed that no hemorrhage occurred on the T2w images after RaSP. Combining all these results and comparisons, it is more likely that the MBs mainly presented stable cavitation in our RaSP experiments. However, considering the lower frequency used in our study, we believe that the acoustic pressure and $M B$ parameters can be further optimized in future explorations when sufficient animal models are available.

The monkeys' blood oxygen levels, respiration rates, and heart rates were stable in all experiments. With MRI detection, hemorrhage was occasionally present after LP10. Under the same acoustic pressure, the LP10 has a higher risk of adverse effects such as hemorrhage or edema. Interestingly, hemorrhage was only found in the G5 group (LP10 after RaSP30) but not in the G3 group (LP10 only), suggesting that repeated ultrasound sonication at the same location to induce BBB opening in a short time interval has a higher risk. Adverse effect accumulation may explain the hemorrhage. The exact reason for this finding is unknown and will be explored in future studies.

We acknowledge that our study has limitations. First, the number of trials and experimental animals were relatively limited. Only 10 trials on 2 monkeys were performed in this study. However, based on the observations from the 2 monkeys, these results are comparable and consistent between the trials with the same ultrasound protocol for each monkey. Second, the focal area of our transducer was relatively large for targeted BBB opening. Using a transducer with a higher frequency, electronic steering for skull correction using a phased array will be considered in future studies to avoid the focal point distortion. Third, only the ultrasound burst length varied, while other ultrasound and MB parameters were kept the same. Studies are needed with varying acoustic power, burst lengths, and $\mathrm{MB}$ parameters, including size and dose, to find the optimized parameters for shorter BBB recovery time. Fourth, the duration of BBB opening after RaSP and drug delivery efficiency in primates, need further quantitative studies.

\section{Conclusions}

This work demonstrated the feasibility and safety of RaSP ultrasound to open the BBB in a non-human primate model. The relative signal enhancement in RaSP30 and RaSP60 reached more than $30 \%$ or $60 \%$ of that with LP sonication in our experiments, respectively. Furthermore, the energy deposition in RaSP30 and RaSP60 was only $3 \%$ and $6 \%$ of LP, respectively. The results suggest that opening the BBB using RaSP is preferable to opening with traditional LPs. Measures to shorten the duration of $\mathrm{BBB}$ opening after RaSP ultrasound should be investigated in future experiments.

\section{Acknowledgments}

The authors thank Mr. De Meng, Mr. Changjun Tie, Professor Lijuan Zhang, Dr. Lili Niu, and Dr. Zonghai 
Sheng for their help.

Funding: This research was supported by the National Natural Science Foundation of China (No. 81527901, 81801724 and 61901462), the Guangdong Grant for Key Technologies for Treatment of Brain Disorders (No. 2018B030332001), the Scientific Instrument Innovation Team of the Chinese Academy of Sciences (No. GJJSTD20180002), the International Partnership Program of Chinese Academy of Sciences (Grant No. 154144KYSB20180063), the Shenzhen Double Chain Grant [2018] 256, and the Key Technology and Equipment R\&D Program of Major Science and Technology Infrastructure of Shenzhen: ZDKJ20190204004 and ZDKJ20190204003.

\section{Footnote}

Conflicts of Interest: All authors have completed the ICMJE uniform disclosure form (available at http://dx.doi. org/10.21037/qims-20-1047). The authors have no conflicts of interest to declare.

Ethical Statement: All animal procedures were approved by the Institutional Ethical Committee of Animal Experimentation of Shenzhen Institutes of Advanced Technology, Chinese Academy of Sciences. The experiments (Certificate number: original-SIAT-IRB-170425-YGSZHR-A0094-2, amended-SIAT-IACUC-20200417ZKYSZXJJSYJY-YB-ZHR-2-04) were carried out strictly in accordance with governmental and international guidelines on animal experimentation. All efforts were made to minimize the number of animals used and their suffering during experiments, according to the request of Biosafety and Animal Ethics

Open Access Statement: This is an Open Access article distributed in accordance with the Creative Commons Attribution-NonCommercial-NoDerivs 4.0 International License (CC BY-NC-ND 4.0), which permits the noncommercial replication and distribution of the article with the strict proviso that no changes or edits are made and the original work is properly cited (including links to both the formal publication through the relevant DOI and the license). See: https://creativecommons.org/licenses/by-nc-nd/4.0/.

\section{References}

1. Meng Y, Pople CB, Lea-Banks H, Abrahao A, Davidson B, Suppiah S, Vecchio LM, Samuel N, Mahmud F, Hynynen
K, Hamani C, Lipsman N. Safety and efficacy of focused ultrasound induced blood-brain barrier opening, an integrative review of animal and human studies. J Control Release 2019;309:25-36.

2. Pardridge WM. The blood-brain barrier: bottleneck in brain drug development. NeuroRx 2005;2:3-14.

3. Wong KH, Riaz MK, Xie YN, Zhang X, Liu Q, Chen HJ, Bian ZX, Chen XY, Lu AP, Yang ZJ. Review of Current Strategies for Delivering Alzheimer's Disease Drugs across the Blood-Brain Barrier. Int J Mol Sci 2019;20:381.

4. Bystritsky A, Korb AS, Douglas PK, Cohen MS, Melega WP, Mulgaonkar AP, DeSalles A, Min BK, Yoof SS. A review of low-intensity focused ultrasound pulsation. Brain Stimul 2011;4:125-36.

5. Beccaria K, Canney M, Bouchoux G, Puget S, Grill J, Carpentier A. Blood-brain barrier disruption with lowintensity pulsed ultrasound for the treatment of pediatric brain tumors: a review and perspectives. Neurosurg Focus 2020;48:E10.

6. Lee EJ, Fomenko A, Lozano AM. Magnetic ResonanceGuided Focused Ultrasound: Current Status and Future Perspectives in Thermal Ablation and Blood-Brain Barrier Opening. J Korean Neurosurg Soc 2019;62:10-26.

7. Hynynen K, McDannold N, Vykhodtseva N, Jolesz FA. Noninvasive MR imaging-guided focal opening of the blood-brain barrier in rabbits. Radiology 2001;220:640-6.

8. McDannold N, Vykhodtseva N, Raymond S, Jolesz FA, Hynynen K. MRI-guided targeted blood-brain barrier disruption with focused ultrasound: Histological findings in rabbits. Ultrasound Med Biol 2005;31:1527-37.

9. Samiotaki G, Vlachos F, Tung YS, Konofagou EE. A quantitative pressure and microbubble-size dependence study of focused ultrasound-induced blood-brain barrier opening reversibility in vivo using MRI. Magn Reson Med 2012;67:769-77.

10. Downs ME, Buch A, Sierra C, Karakatsani ME, Teichert T, Chen SS, Konofagou EE, Ferrera VP. Long-Term Safety of Repeated Blood-Brain Barrier Opening via Focused Ultrasound with Microbubbles in Non-Human Primates Performing a Cognitive Task. PLoS One 2015;10:e125911.

11. Lipsman N, Meng Y, Bethune AJ, Huang YX, Lam B, Masellis M, Herrmann N, Heyn C, Aubert I, Boutet A, Smith GS, Hynynen K, Black SE. Blood-brain barrier opening in Alzheimer's disease using MR-guided focused ultrasound. Nat Commun 2018;9:2336.

12. Mainprize T, Lipsman N, Huang Y, Meng Y, Bethune A, Ironside S, Heyn C, Alkins R, Trudeau M, Sahgal A, Perry 
J, Hynynen K. Blood-Brain Barrier Opening in Primary Brain Tumors with Non-invasive MR-Guided Focused Ultrasound: A Clinical Safety and Feasibility Study. Sci Rep 2019;9:321.

13. Idbaih A, Canney M, Belin L, Desseaux C, Vignot A, Bouchoux G, Asquier N, Law-Ye B, Leclercq D, Bissery A, De Rycke Y, Trosch C, Capelle L, Sanson M, Hoang-Xuan K, Dehais C, Houillier C, Laigle-Donadey F, Mathon B, Andre A, Lafon C, Chapelon JY, Delattre JY, Carpentier A. Safety and Feasibility of Repeated and Transient Blood-Brain Barrier Disruption by Pulsed Ultrasound in Patients with Recurrent Glioblastoma. Clin Cancer Res 2019;25:3793-801.

14. Kamimura HA, Flament J, Valette J, Cafarelli A, Aron Badin R, Hantraye P, Larrat B. Feedback control of microbubble cavitation for ultrasound-mediated bloodbrain barrier disruption in non-human primates under magnetic resonance guidance. J Cereb Blood Flow Metab 2019;39:1191-203.

15. McDannold N, Vykhodtseva N, Hynynen K. Effects of acoustic parameters and ultrasound contrast agent dose on focused-ultrasound induced blood-brain barrier disruption. Ultrasound Med Biol 2008;34:930-7.

16. Todd N, Angolano C, Ferran C, Devor A, Borsook D, McDannold N. Secondary effects on brain physiology caused by focused ultrasound-mediated disruption of the blood-brain barrier. J Control Release 2020;324:450-9.

17. Chai WY, Chu PC, Tsai MY, Lin YC, Wang JJ, Wei KC, Wai YY, Liu HL. Magnetic-resonance imaging for kinetic analysis of permeability changes during focused ultrasound-induced blood-brain barrier opening and brain drug delivery. J Control Release 2014;192:1-9.

18. Kovacs ZI, Kim S, Jikaria N, Qureshi F, Milo B, Lewis BK, Bresler M, Burks SR, Frank JA. Disrupting the blood-brain barrier by focused ultrasound induces sterile inflammation. Proc Natl Acad Sci U S A 2017;114:E75-E84.

19. Choi JJ, Selert K, Gao ZM, Samiotaki G, Baseri B, Konofagou EE. Noninvasive and localized blood-brain barrier disruption using focused ultrasound can be achieved at short pulse lengths and low pulse repetition frequencies. J Cereb Blood Flow Metab 2011;31:725-37.

20. O'Reilly MA, Waspe AC, Ganguly M, Hynynen K. Focused-Ultrasound Disruption of the Blood-Brain Barrier Using Closely-Timed Short Pulses: Influence of Sonication Parameters and Injection Rate. Ultrasound Med Biol 2011;37:587-94.

21. Samiotaki G, Konofagou EE. Dependence of the reversibility of focused- ultrasound-induced blood-brain barrier opening on pressure and pulse length in vivo. IEEE Trans Ultrason Ferroelectr Freq Control 2013; 60:2257-65.

22. Morse SV, Pouliopoulos AN, Chan TG, Copping MJ, Lin JL, Long NJ, Choi JJ. Rapid Short-pulse Ultrasound Delivers Drugs Uniformly across the Murine BloodBrain Barrier with Negligible Disruption. Radiology 2019;291:459-66.

23. Mórocz IA, Hynynen K, Gudbjartsson H, Peled S, Colucci V, Jolesz FA. Brain edema development after MRI-guided focused ultrasound treatment. J Magn Reson Imaging 1998;8:136-42.

24. Hermier M, Nighoghossian N, Derex L, Berthezene Y, Blanc-Lasserre K, Trouillas P, Froment JC. MRI of acute post-ischemic cerebral hemorrhage in stroke patients: diagnosis with T2*-weighted gradient-echo sequences. Neuroradiology 2001;43:809-15.

25. Horodyckid C, Canney M, Vignot A, Boisgard R, Drier A, Huberfeld G, Francois C, Prigent A, Santin MD, Adam C, Willer JC, Lafon C, Chapelon JY, Carpentier A. Safe longterm repeated disruption of the blood-brain barrier using an implantable ultrasound device: a multiparametric study in a primate model. J Neurosurg 2017;126:1351-61.

26. Hynynen K, McDannold N, Sheikov NA, Jolesz FA, Vykhodtseva N. Local and reversible blood-brain barrier disruption by noninvasive focused ultrasound at frequencies suitable for trans-skull sonications. Neuroimage 2005;24:12-20.

27. Karakatsani ME, Samiotaki G, Downs ME, Ferrera VP, Konofagou EE. Targeting Effects on the Volume of the Focused Ultrasound-Induced Blood-Brain Barrier Opening in Nonhuman Primates In Vivo. IEEE Trans Ultrason Ferroelectr Freq Control 2017;64:798-810.

28. Samiotaki G, Karakatsani ME, Buch A, Papadopoulos S, Wu SY, Jambawalikar S, Konofagou EE. Pharmacokinetic analysis and drug delivery efficiency of the focused ultrasound-induced blood-brain barrier opening in nonhuman primates. Magn Reson Imaging 2017;37:273-81.

29. Marquet F, Teichert T, Wu SY, Tung YS, Downs M, Wang S, Chen C, Ferrera V, Konofagou EE. Real-time, transcranial monitoring of safe blood-brain barrier opening in non-human primates. PLoS One 2014;9:e84310.

30. Sun T, Samiotaki G, Wang S, Acosta C, Chen CC, Konofagou EE. Acoustic cavitation-based monitoring of the reversibility and permeability of ultrasound-induced blood-brain barrier opening. Phys Med Biol 2015; 60:9079-94.

31. O'Reilly MA, Hough O, Hynynen K. Blood-BrainBarrier 
Closure TimeAfter Controlled Ultrasound-Induced Opening Is Independent of Opening Volume. J Ultrasound Med 2017;36:475-83.

32. McDannold N, Vykhodtseva N, Hynynen K. Bloodbrain barrier disruption induced by focused ultrasound and circulating preformed microbubbles appears to be characterized by the mechanical index. Ultrasound Med Biol 2008;34:834-40.

33. Wu SY, Tung YS, Marquet F, Downs ME, Sanchez

Cite this article as: Zhou H, Liu Y, Long X, Qiao Y, Lee J, Liu X, Zheng H, Zou C. MR-guided blood-brain barrier opening induced by rapid short-pulse ultrasound in non-human primates. Quant Imaging Med Surg 2021;11(6):2415-2427. doi: 10.21037/qims-20-1047
CS, Chen CC, Ferrera V, Konofagou E. Transcranial Cavitation Detection in Primates During Blood-Brain

Barrier Opening-A Performance Assessment Study. IEEE Trans Ultrason Ferroelectr Freq Control 2014;61:966-78.

34. Arvanitis CD, Livingstone MS, Vykhodtseva N, McDannold N. Controlled Ultrasound-Induced BloodBrain Barrier Disruption Using Passive Acoustic Emissions Monitoring. PLoS One 2012;7:e45783. 
(A)

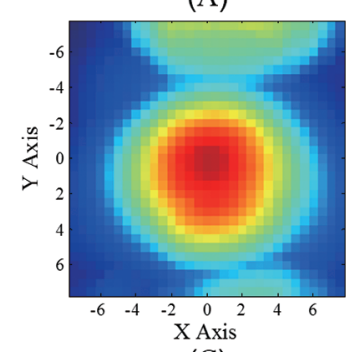

(C)

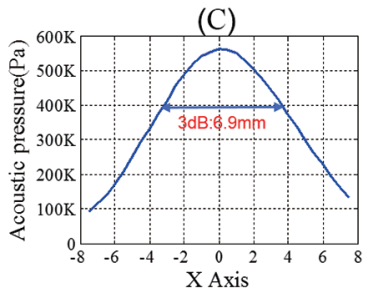

(B)

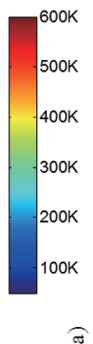

$500 \mathrm{~K}$
$500 \mathrm{~K}$
$300 \mathrm{~K}$
$200 \mathrm{~K}$
$100 \mathrm{~K}$

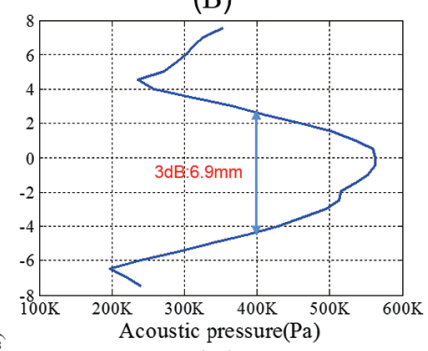

(D)

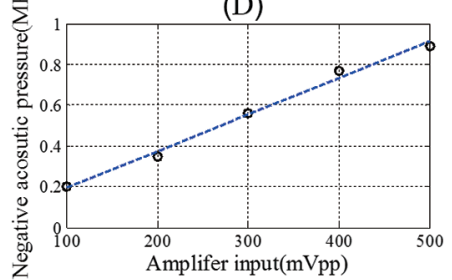

(E)

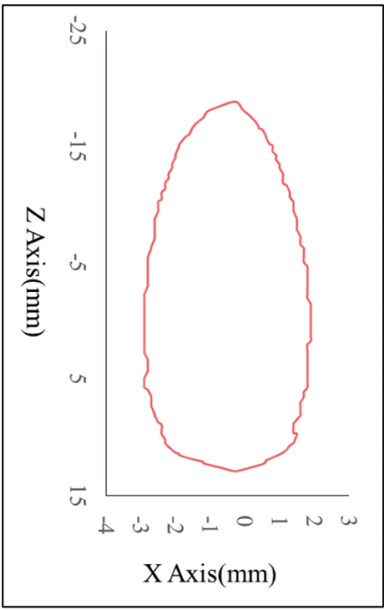

Figure S1 The acoustic field characterization of the custom-built transducer in water. (A) 2D acoustic power distribution in the focal XY plane. (B,C) The acoustic power distribution along the $\mathrm{X}$ axis and $\mathrm{Y}$ axis when the amplifier was driven by $300 \mathrm{mVpp}$ input. (D) Negative acoustic pressure measured by a hydrophone at different input voltages of the amplifier. (E) $3 \mathrm{~dB}$ contour line of acoustic power intensity in the focal $\mathrm{XZ}$ plane, the length on the $\mathrm{Z}$ axis is $32 \mathrm{~mm}$.

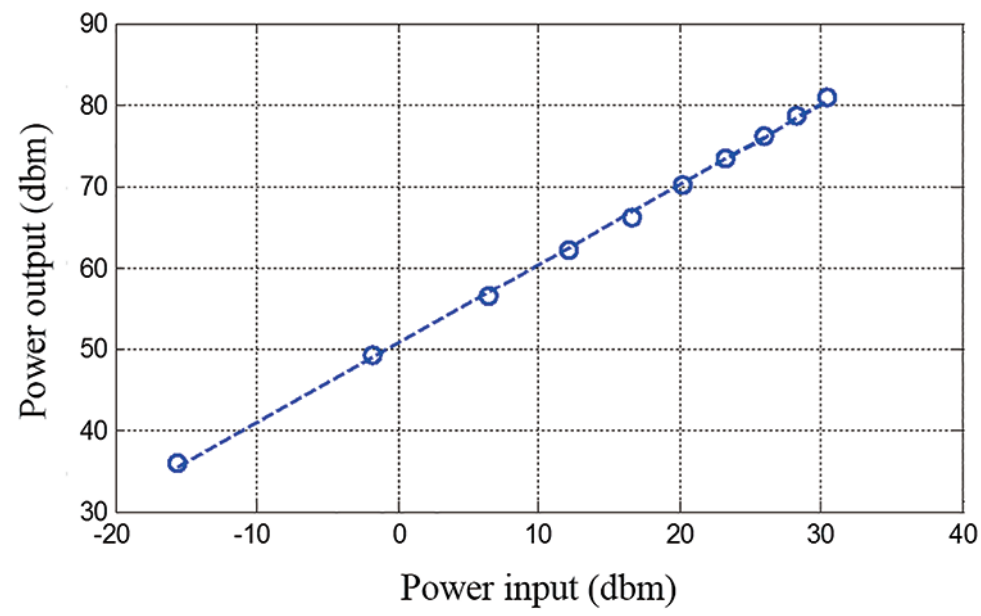

Figure S2 The gain of the custom-built power amplifier was measured as $\sim 50 \mathrm{~dB}$ operating at $300 \mathrm{kHz}$. 


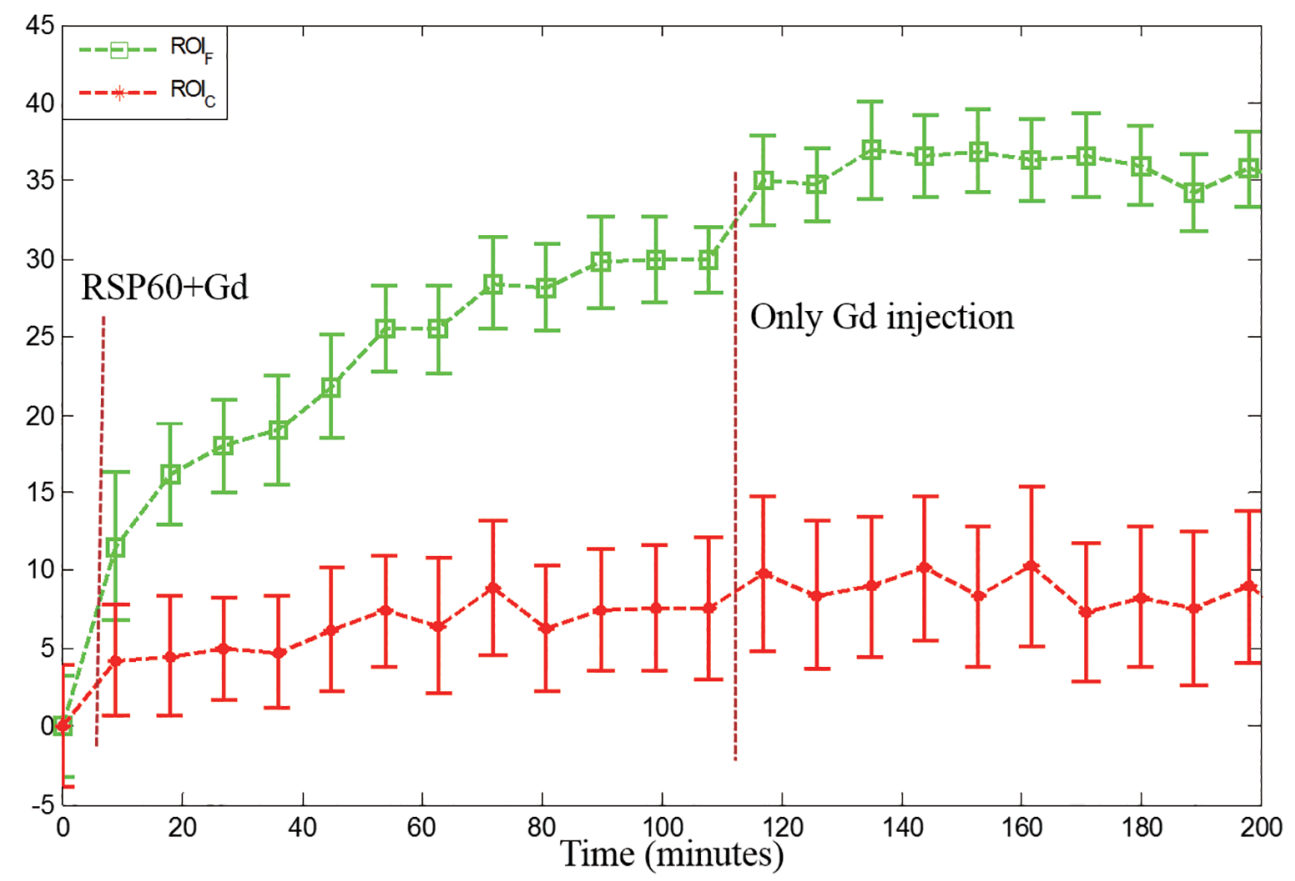

Figure S3 To verify whether the BBB was closed 100 minutes after RSP60 sonication, Gd was injected again 100 minutes after RSP60. The signal intensity in ROIF continued to increase after the second contrast injection even without ultrasound, while the intensity in ROIC was almost the same. This observation indicates that the BBB was not closed 100 minutes after RSP60.

Table S1 The relative signal enhancement (\%) in the experimental groups

\begin{tabular}{|c|c|c|c|c|c|c|c|c|}
\hline $\begin{array}{l}\text { Group } \\
\text { Protocol }\end{array}$ & & $\frac{\mathrm{G} 1}{\mathrm{RaSP} 30}$ & $\frac{\mathrm{G} 2}{\mathrm{RaSP} 60}$ & $\frac{\text { G3 }}{\text { LP10 }}$ & \multicolumn{2}{|c|}{ G4 } & \multicolumn{2}{|c|}{ G5 } \\
\hline \multirow{2}{*}{ M21 } & $\mathrm{ROI}_{\mathrm{C}}$ & $6.4 \pm 3.7$ & $8.9 \pm 4.0$ & $10.0 \pm 2.6$ & $4.6 \pm 3.2$ & $8.1 \pm 3.3$ & $5.9 \pm 4.3$ & $8.6 \pm 3.5$ \\
\hline & Diff & 6.3 & 21.6 & 35.7 & 8.6 & 24.4 & 7.4 & 38.6 \\
\hline \multirow{2}{*}{ M22 } & $\mathrm{ROI}_{\mathrm{C}}$ & $4.5 \pm 3.8$ & $5.5 \pm 6.7$ & $6.9 \pm 4.5$ & $3.0 \pm 3.5$ & $7.6 \pm 3.5$ & $7.5 \pm 6.2$ & $8.7 \pm 5.8$ \\
\hline & Diff & 9.3 & 18.2 & 26.1 & 10.1 & 18.5 & 5.1 & 28.9 \\
\hline
\end{tabular}

$\mathrm{ROI}_{\mathrm{F}}$ : region of interest at focus. $\mathrm{ROI}_{\mathrm{C}}$ : region of interest at the opposite hemisphere for comparison. Diff: the difference between relative signal enhancement of $\mathrm{ROI}_{\mathrm{F}}$ and $\mathrm{ROI}_{\mathrm{C}}$. 